\title{
Filamentous fungi from extreme environments as a promising source of novel bioactive secondary metabolites
}

OPEN ACCESS

Edited by:

Ana Lúcia Leitão,

Universidade Nova de Lisboa,

Portugal

Reviewed by:

Charilaos Xiros,

Bern University of Applied Sciences,

Switzerland

Jean Marie François,

Laboratoire d'Ingenierie des

Systèmes Biologiques et procédésn

UMR -CNRS 5504, France

${ }^{*}$ Correspondence:

Francisco Fierro,

División de Ciencias Biológicas y de la

Salud, Departamento

de Biotecnología, Universidad

Autónoma Metropolitana-Unidad

Iztapalapa, Avenida San Rafael

Atlixco 186, Colonia Vicentina,

México D.F. 09340, Mexico

fierrof@xanum.uam.mx;

Inmaculada Vaca,

Facultad de Ciencias, Departamento

de Química, Universidad

de Chile, Las Palmeras 3425, Ñuñoa,

Santiago 7800024, Chile

inmavaca@uchile.cl

Specialty section:

This article was submitted to

Microbial Physiology and Metabolism,

a section of the journal

Frontiers in Microbiology

Received: 02 June 2015

Accepted: 17 August 2015

Published: 09 September 2015

Citation:

Chávez R, Fierro F, García-Rico RO and Vaca I (2015) Filamentous fungi

from extreme environments as a promising source of novel bioactive

secondary metabolites.

Front. Microbiol. 6:903.

doi: 10.3389/fmicb.2015.00903

\begin{abstract}
Renato Chávez ${ }^{1}$, Francisco Fierro ${ }^{2 *}$, Ramón O. García-Rico ${ }^{3}$ and Inmaculada Vaca ${ }^{4 *}$
' Facultad de Química y Biología, Departamento de Biología, Universidad de Santiago de Chile, Santiago, Chile, ${ }^{2}$ División de Ciencias Biológicas y de la Salud, Departamento de Biotecnología, Universidad Autónoma Metropolitana-Unidad Iztapalapa, México D.F., Mexico, ${ }^{3}$ Grupo GIMBIO, Facultad de Ciencias Básicas, Departamento de Microbiología, Universidad de Pamplona, Pamplona, Colombia, ${ }^{4}$ Facultad de Ciencias, Departamento de Química, Universidad de Chile, Santiago, Chile
\end{abstract}

Natural product search is undergoing resurgence upon the discovery of a huge previously unknown potential for secondary metabolite (SM) production hidden in microbial genomes. This is also the case for filamentous fungi, since their genomes contain a high number of "orphan" SM gene clusters. Recent estimates indicate that only $5 \%$ of existing fungal species have been described, thus the potential for the discovery of novel metabolites in fungi is huge. In this context, fungi thriving in harsh environments are of particular interest since they are outstanding producers of unusual chemical structures. At present, there are around 16 genomes from extreme environment-isolated fungi in databases. In a preliminary analysis of three of these genomes we found that several of the predicted SM gene clusters are probably involved in the biosynthesis of compounds not yet described. Genome mining strategies allow the exploitation of the information in genome sequences for the discovery of new natural compounds. The synergy between genome mining strategies and the expected abundance of SMs in fungi from extreme environments is a promising path to discover new natural compounds as a source of medically useful drugs.

\footnotetext{
Keywords: natural products, filamentous fungi, secondary metabolites, extreme environments, genome mining, metagenomics
}

\section{Introduction}

The last decades of the 20th century witnessed a decline in the search for natural compounds as pharmaceutically useful drugs. A number of reasons accounted for this trend (Strohl, 2000). However, natural products present key advantages over synthesized chemicals which make them an unsubstitutable source of potential new drugs. They show greater structural diversity as compared to chemical synthetic compounds. This diversity makes them a potentially infinite source of chemical diversity, and as a consequence they present a wide range of biological activities (Demain, 2014). Natural products or compounds derived from them constituted over $35 \%$ of approved and pre-new drug application candidates in the period spanning 1981-2010, and 48.6\% in the case of anti-cancer drugs since the 1940s (Newman and Cragg, 2012).

Out of about 1 million natural products, $\sim 25 \%$ are biologically active. Of these, about $60 \%$ come from plants, and most of the rest from microbes. In the microbial world, fungi stand out as the most prolific producers of bioactive compounds, with $\sim 42 \%$ of the total (Demain, 2014). 
Classical methodologies for the discovery of microbial natural products mostly relied in the cultivation of isolates in the laboratory followed by a bioactivity-guided fractionation and identification of the purified compounds. The arrival of the genomic era meant a radical change in the approach to discover new natural products. The analysis of microbial genomes made evident that they contain numerous genes likely to participate in the biosynthesis of structurally complex compounds, but which are not associated with the production of known metabolites. This phenomenon was first recognized in strains from Streptomyces (Omura et al., 2001; Bentley et al., 2002) and was soon replicated in filamentous fungi (Keller et al., 2005). Nowadays, it is acknowledged that fungi have a potential of secondary metabolite (SM) production far beyond the number of compounds currently known. Genome mining strategies allow the exploitation of the information provided by genome sequences for the discovery of new natural compounds. This wealth of genomic information is aided by improvements in chemical analytical methodologies, which allow higher separation capacity and sensitivity (Molinski, 2010; Li et al., 2015). Thus a resurgence of natural product discovery is underway, and fungi from extreme environments may make a significant contribution to it.

In this article we will discuss why fungi isolated from extreme environments are an excellent potential source of new natural products with novel and/or unusual chemical structures, and will propose some genome and metagenome mining methodologies which can be successfully applied to them.

\section{Are Fungi from Extreme Environments Good Potential Producers of Novel Secondary Metabolites?}

Each fungal species has its own chemotype, and thus an effective strategy to find new compounds is to study the whole secondary metabolome of new species. The chances of isolating new fungal species are greater if the samples come from non-mesophilic environments, such as those characterized by high salinity, high radiation, limited nutrients, extreme temperatures and pressures and variable acidity. Organisms from these extreme habitats have developed survival strategies for growing and reproducing in such harsh conditions, among them the production of small organic molecules with specific biological activities, for example, cryoprotectant molecules such as sugars and polyols to stabilize membranes and maintain turgor pressure (Timling and Taylor, 2012), osmotically active compounds such as polyols in xerotolerant fungi (Gunde-Cimerman and Zalar, 2014), and fungal melanins for protection against freezing and UV radiation (Nosanchuk and Casadevall, 2003). Since fungi from extreme habitats are subjected to environmental conditions not met by mesophilic fungi, it is expected that many of the compounds they synthesize are specific to them.

Fungi from extreme environments seem therefore good potential candidates for the isolation of new bioactive compounds, and this interest has been reflected in the gradual increase of published articles reporting new compounds from these fungi. Supplementary Table S1 summarizes the compounds isolated from fungi growing in extreme environments reported since 2004. Although the number of studies is still low, a remarkable percentage of them (around 40\%) report the isolation of new natural products with unusual structural features, for instance the spiromastixones $\mathrm{A}-\mathrm{O}$, a group of depsidonebased derivatives from Spiromastix sp. (isolated from deep sea sediments of the South Atlantic Ocean; Niu et al., 2014) which contain $n$-propyl substituents in their structure, a feature rarely found in natural products, and the four asterric acid derivatives bearing the unusual nitro group found in the Antarctic fungus Pseudogymnoascus sp. (Figueroa et al., 2015). This percentage compares favorably with what is usually found in mesophilic fungi; in a search we performed on studies published since 2004 describing new compounds from a typical mesophilic SM producer group, Aspergillus section Nigri, we found that only in $13 \%$ of the studies compounds showing unusual, previously undescribed, structures were reported. Therefore, there seems to be sufficient evidence that fungi isolated from extreme environments have an excellent potential as producers of unusual compounds with unique structures.

\section{How to Maximize Natural Products Discovery from Extreme Environment Fungi}

When considering strategies for the isolation of compounds from fungi growing in extreme environments, several considerations must be taken into account. Firstly, isolates from extreme environments may have individual specific requirements to express their SM pathways which are not easy to find/set in the laboratory; likewise other fungi, many pathways are expected to remain silent under laboratory conditions (see below). Secondly, how many of the total number of fungi living in such environments are we able to cultivate in the laboratory?, and thus how many are unculturable and evade our screenings? Magnuson and Lasure (2002) reported that 70-90\% of fungi in different soil environments were unculturable. Genome mining offers an array of methods to face the first problem, whereas metagenomics can be applicable to widen the scope of our search for bioactive fungal compounds to those produced by unculturable fungi. Both approaches will be discussed in the next sections.

Traditionally, isolation of natural compounds produced by fungi was based on culturing them under standard laboratory conditions. Variations in the conditions and the media have great impact on the profile of SMs produced (Larsen et al., 2005), and thus different OSMAC (one strain-many compounds, Bode et al., 2002) strategies are developed by research groups to induce the synthesis of compounds (e.g., VanderMolen et al., 2013; Hewage et al., 2014). In the particular case of fungi from extreme environments, culture conditions different from the standard ones have been sometimes used in an attempt to recreate environmental characteristics of their habitats. Most of fungi isolated from cold environments are cultured at $15-20^{\circ} \mathrm{C}$, and fungi isolated from saline environments are grown in media with 
$\mathrm{NaCl}$ concentrations of $10 \%$ or above. However, a significant number of the reports in Supplementary Table S1 used standard culture conditions, this is especially noticeable for fungi isolated from deep-sea samples, mostly grown at $28^{\circ} \mathrm{C}$ and atmospheric pressure. The use of conditions very different from those present in the native environments hinders the prospects of finding new compounds from extreme environment fungi.

The analysis of fungal genomes has revealed that the potential number of SMs fungi can produce is much higher than what can be isolated and identified using classical approaches. The main reason that explains why many SMs "encoded" in the genome are not detected seems to be that SM clusters are often silent when fungi are cultured in the laboratory (Brakhage et al., 2008; Hertweck, 2009), a phenomenon that might be still more extensive in fungi from extreme environments. One of the main challenges of genome mining is how to activate these silent clusters so that the "cryptic" compounds they produce can be isolated. Epigenetic regulation has been pointed as the main mechanism causing SM gene cluster silencing (Reyes-Domínguez et al., 2010; Strauss and Reyes-Domínguez, 2011). A general strategy for activation of silent clusters is the use of heterochromatin remodeling compounds, such as 5-azacytidine (DNA methyltransferase inhibitor), trichostatine A or suberoylanilide hydroxamic acid (histone deacetylase inhibitors). Several reports describe that addition of these inhibitors to culture media causes changes in the expression profile of SM gene clusters (Lin et al., 2013), the profile of metabolites detected in the broths (Williams et al., 2008; Chung et al., 2013), and has allowed the isolation of previously unknown compounds, for example nygerone A from Aspergillus niger (Henrikson et al., 2009) and new sesquiterpenoids from Aspergillus sydowii (Chung et al., 2013). Since this strategy does not require sequencing of the genome, it may be suitable for initial metabolite screenings of extreme environment fungi, in combination with OSMAC strategies.

\section{Genome Mining and Its Application to the Discovery of New Metabolites from Extreme Environment-Isolated Fungi}

Genome mining requires an extensive amount of genome sequence information in order to explore in-depth the SM biosynthetic potential of a microorganism. Many of the SM gene clusters are expected to remain silent when extreme environment fungi are cultured in the laboratory, and therefore identifying these clusters is a key step to exploit this potential. For new isolates, top-down approaches (OSMAC-based strategies in combination with the use of epigenetic modifiers) can be useful to select the best candidates for more in-depth analysis, and may even result in the production and identification of some interesting compounds (Bode et al., 2002; Wang et al., 2014). Isolates showing the best potential capacity for production of new compounds can then be selected for genome analysis. The increasing number of facilities for whole genome sequencing will allow that more specimens can be analyzed in this manner in a near future.
At present, there are some 515 completed or in progress fungal genomes in public databases, of which around 16 belong to fungi isolated from extreme environments. As a preliminary assessment to test if fungi from extreme environments can be a valuable source of novel SMs we scanned the genomes of three fungi, the cold-loving Pseudogymnoascus pannorum and $P$. destructans and the thermophilic Thermomyces lanuginosus. We used antiSMASH, a software designed to systematically predict clustered SM genes based on their genomic context and domain content (Blin et al., 2013; Weber et al., 2015). The results of this analysis are summarized in Table 1. When compared to a model fungus, Aspergillus nidulans, the three analyzed fungi show an interesting potential as producers of novel SMs. They have a similar number of SM clusters (14-20) and, interestingly, no more than three of them (depending on the species) are homologous to previously described clusters with known function, that is, the compound they putatively synthesize has been identified in other organisms. Also remarkable is the fact that although several clusters in the extreme environment fungi present partial synteny with clusters from other genomes, they possess 1-3 clusters that seem to be unique, since they have no significant homology to any other genomic sequence in databases. In comparison, all A. nidulans clusters have homologs in other organisms. These unique clusters are likely to be involved in the synthesis of novel compounds, possibly with novel/unusual chemical structures.

There is an important difference in the number of SM clusters found with antiSMASH between the extreme environment fungi and $A$. nidulans. Taking into account that their genome sizes are similar (although that of $T$. lanuginosus is $23 \%$ smaller than the A. nidulans one), this difference is somewhat striking. Biological differences might account for this discordance, they are all Ascomycota, but from different lineages. Pseudogymnoascus spp. belongs to the class Leotiomycetes, whereas A. nidulans and T. lanuginosus are Eurotiales. Another plausible explanation is that results are biased by the way of working of antiSMASH. This software compares the predicted proteins in a DNA sequence against a curated collection of profile hidden Markov models ( $\mathrm{pHMM}$ ) that describe sequences of key biosynthetic enzymes of known classes of SMs. Since pHMM are probabilistic models built from an aligned set of representative sequences, these models can be biased by the sequences used in their construction. Genomes from mesophilic fungus such $A$. nidulans are the most abundant in databases, and therefore, they were probably intensively used in the generation of these $\mathrm{pHMM}$. Therefore, analyses performed with these $\mathrm{pHMM}$ will easily find clusters showing similarity to those from mesophilic organisms, but may be less proficient to find novel enzymes of novel SM pathways from extreme environment fungi. Other bioinformatics tools for SM gene search are also available (Khaldi et al., 2010; Fedorova et al., 2012; Boddy, 2014), and the combined capacities of these tools may yield improved results.

Once a new SM cluster has been identified, the next step is isolating and identifying the metabolite/s it synthesizes, this is one of the bottlenecks of genome mining. The structure of the cluster, the filiation of the genes and the domains present in the encoded enzymes may offer clues about the chemical features 
TABLE 1 | Summary of SM clusters found by antiSMASH in some fungal genomes.

\begin{tabular}{|c|c|c|c|c|c|c|}
\hline Organism & $\begin{array}{l}\text { GenBank accession } \\
\text { number of the } \\
\text { genome }\end{array}$ & $\begin{array}{l}\text { Environmental } \\
\text { characteristic }\end{array}$ & $\begin{array}{l}\text { Genome } \\
\text { size (Mbp) }\end{array}$ & $\begin{array}{l}\text { Number of } \\
\text { clusters found by } \\
\text { antiSmash }\end{array}$ & $\begin{array}{l}\text { Number of clusters } \\
\text { homologous to clusters } \\
\text { with known function (\%) }\end{array}$ & $\begin{array}{l}\text { Number of clusters } \\
\text { without homologs in } \\
\text { other genomes (\%) }\end{array}$ \\
\hline Aspergillus nidulans & $\begin{array}{l}\text { BN001301; BN001302; } \\
\text { BN001303; BN001304; } \\
\text { BN001305; BN001306; } \\
\text { BN001307; BN001308 }\end{array}$ & $\begin{array}{l}\text { Mesophilic (model) } \\
\text { fungus }\end{array}$ & 31 & 58 & $12(20.7)$ & $0(0)$ \\
\hline $\begin{array}{l}\text { Pseudogymnoascus } \\
\text { destructans }\end{array}$ & AEFC00000000 & Cold-loving fungus & 30.5 & 14 & 2 (14.3) & $1(7.1)$ \\
\hline $\begin{array}{l}\text { Pseudogymnoascus } \\
\text { pannorum }\end{array}$ & AYKR00000000 & Cold-loving fungus & 29.5 & 20 & $3(15.0)$ & $2(10.0)$ \\
\hline $\begin{array}{l}\text { Thermomyces } \\
\text { lanuginosus }\end{array}$ & ANHP00000000 & Thermophilic fungus & 23.3 & 17 & $2(11.8)$ & $3(17.6)$ \\
\hline
\end{tabular}

of the corresponding metabolite, and thus directed separation and analytical strategies can be applied for its identification, an approach that has yielded successful results for the isolation of aspoquinolones A-D and PKS-NRPS hybrid compounds from A. nidulans (Scherlach and Hertweck, 2006; Bergmann et al., 2007). A refinement of this approach is the so-called genomisotopic approach, which makes use of isotope-labeled molecules predicted by bioinformatics to guide the purification process (Gross et al., 2007).
A second major bottleneck is the fact that many SM clusters remain silent even when submitted to different culture conditions. Several reviews on genome mining have been published dealing with the issue of activation of silent clusters and identification of their metabolites (Scherlach and Hertweck, 2009; Brakhage and Schroeckh, 2011; Chiang et al., 2014; Luo et al., 2014; Wiemann and Keller, 2014). Basically, the methodologies described can be divided in two groups, (1) those aiming to activate clusters in the host strain, for instance by using genes

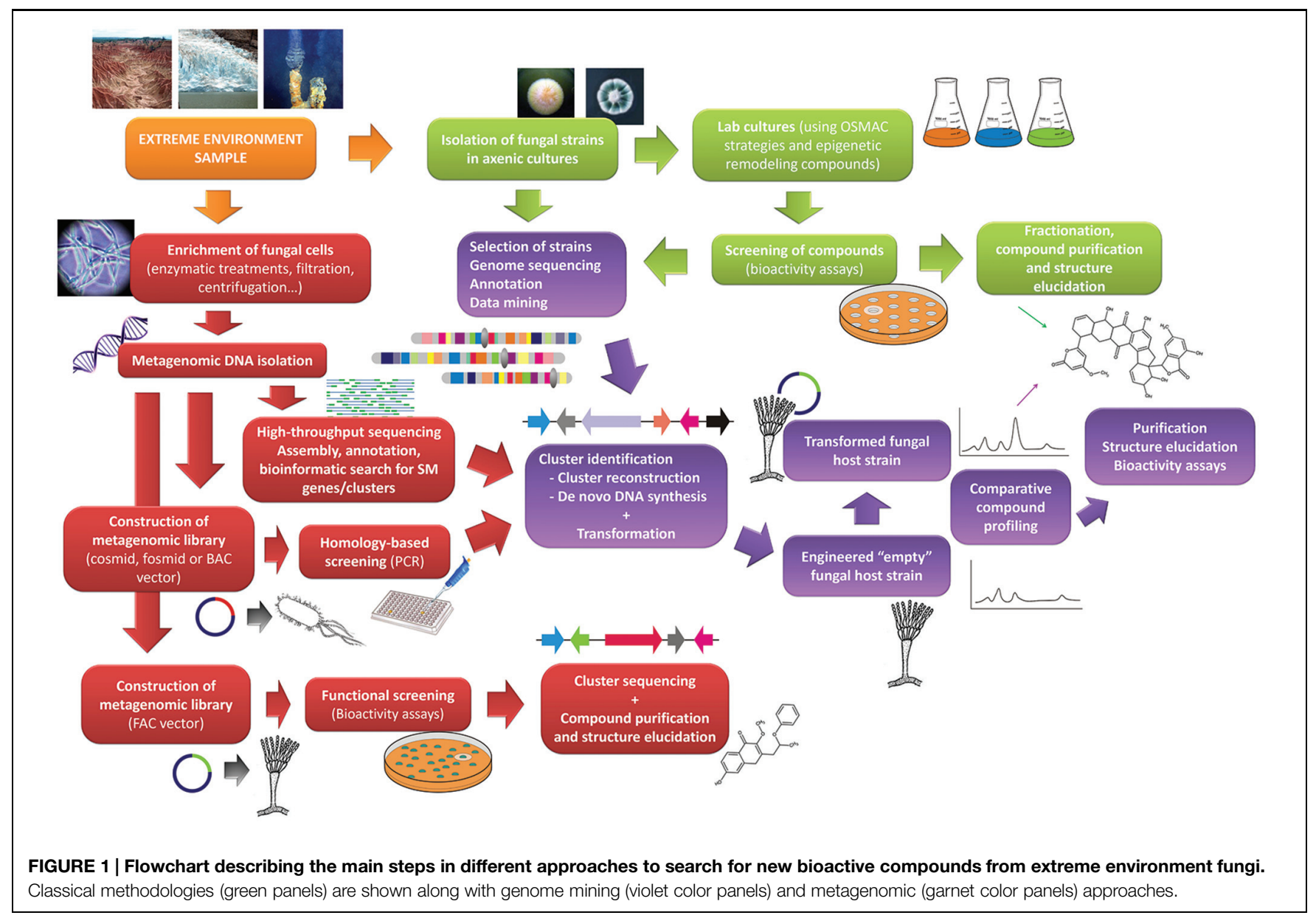


involved in epigenetic regulation (Strauss and Reyes-Domínguez, 2011) or by overexpressing a specific cluster transcriptional regulator (Bromann et al., 2012), and (2) those making use of heterologous expression. In the case of extreme environment fungi, molecular tools are not readily available to apply most of the strategies lying in the first group, except for the utilization of chromatin remodeling compounds mentioned above. Thus the strategy of choice will be the heterologous expression of the cluster in an appropriate host (Figure 1). We enter here in the field of synthetic biology. Different strategies have been developed to reconstruct a whole SM cluster for transferring into a recipient host (reviewed in Lazarus et al., 2014; Anyaogu and Mortensen, 2015). Yeast recombinational cloning has been utilized to clone entire gene clusters and transform A. nidulans with them (Yin et al., 2013). Another possibility is the use of fusion PCR to reconstruct a cluster and then transfer it to the host strain (Chiang et al., 2013). This latter work used an engineered $A$. nidulans strain deficient in its own SM clusters, which facilitates the structure elucidation of the heterologous compound. Other modified recipient strains for heterologous expression of SM clusters have recently been developed (Richter et al., 2014; Kakule et al., 2015). The improvement and increasing affordability of techniques for synthesis of long stretches of DNA (Bang and Church, 2008; Carlson, 2009) will facilitate synthesis of entire clusters, including designed clusters with host promoters and regulatory elements for better gene expression.

\section{Metagenomics for Extreme Environment Fungi in the Search for New Bioactive Compounds}

A comprehensive study on the potential of fungi from extreme environments to produce new bioactive compounds would be incomplete if we did not take into account the unculturable fungi. Currently we have no reliable information regarding the percentage of unculturable fungi present in extreme environments, as much as we do not have information about the capacity of these fungi to produce SMs in comparison to the culturable ones. However, the presumed abundance of unculturable fungi in different environments (Magnuson and Lasure, 2002), and the success of some published studies in finding bacterial SM clusters from complex DNA samples and isolating specific metabolites after their heterologous expression (Nikolouli and Mossialos, 2011 and references therein; Owen et al., 2015) point to a hidden wealth of fungal metabolites waiting to be found, and the feasibility to retrieve them.

The use of metagenomes for the discovery of new natural products is receiving considerable attention (Nováková and Farkašovskỳ, 2013; Wilson and Piel, 2013; Charlop-Powers et al., 2014). Metagenomes usually comprise DNA from prokaryotic and eukaryotic organisms, but some strategies can be devised to enrich some particular type. Jiao et al. (2006) used an enzymatic treatment with cellulases to enrich bacterial DNA from plant samples. Enrichment strategies for extreme environment fungi may be developed too. Eukaryotic DNA could be enriched using lysozime and murein hydrolases followed by differential centrifugation, and combined with cellulase treatment fungal DNA may result prevalent. From here the usual approaches to study metagenomes can be followed (Figure 1). First the choice of using high-throughput sequencing or constructing metagenomic libraries. In the first case an arduous work of assembly, annotation and bioinformatic analyses follows, but which can produce extensive sequence information to retrieve entire SM cluster sequences. In the second case vectors allowing large DNA inserts (e.g., BACs) would be preferable due to the often big size of SM clusters. Libraries can then be submitted to sequence-based screenings in search for SM clusters. Identified clusters can then be transferred to heterologous hosts as described in the previous section. The recent development of FACs (fungal artificial chromosomes) successfully tested to clone and express entire fungal SM clusters in A. nidulans (Bok et al., 2015) is a powerful tool which will allow functional screening of fungal DNA-containing metagenomes.

An interesting approach to estimate the SM biosynthetic potential of different environments was described by Woodhouse et al. (2013), who used TEFAP (Tag-encoded FLX Amplicon Pyrosequencing) targeting NRPS and PKS genes to determine the identity and diversity of SM biosynthetic genes in a complex sample, finding that Australian marine sponges are a good niche to explore for new natural products.

\section{Conclusion}

Fungi gowing in extreme environments are a potential huge reservoir of novel bioactive natural products. Preliminary search of three genomes from extreme environment fungi revealed the presence of several SM clusters with no significant homology to any other genomic sequence in databases or containing only some genes showing identity to others in databases (Table 1). These clusters are likely to form novel compounds with unusual structures, a feature previously reported in other extreme environment fungi (Supplementary Table S1). Genome and metagenome mining technologies will make it possible to exploit this valuable resource.

\section{Acknowledgments}

This work was supported by grant Fondecyt 1150894 of Chile. RC thanks the support of DICYT-USACH.

\section{Supplementary Material}

The Supplementary Material for this article can be found online at: http://journal.frontiersin.org/article/10.3389/fmicb. 2015.00903 


\section{References}

Anyaogu, D. C., and Mortensen, U. H. (2015). Heterologous production of fungal secondary metabolites in Aspergilli. Front. Microbiol. 6:77. doi: 10.3389/fmicb.2015.00077

Bang, D., and Church, G. M. (2008). Gene synthesis by circular assembly amplification. Nat. Methods 5, 37-39. doi: 10.1038/nmeth1136

Bentley, S. D., Chater, K. F., Cerdeno-Tarraga, A.-M., Challis, G. L., Thomson, N. R., James, K. D., et al. (2002). Complete genome sequence of the model actinomycete Streptomyces coelicolor A3(2). Nature 417, 141-147. doi: $10.1038 / 417141 \mathrm{a}$

Bergmann, S., Schuemann, J., Scherlach, K., Lange, C., Brakhage, A. A., and Hertweck, C. (2007). Genomics-driven discovery of PKS-NRPS hybrid metabolites from Aspergillus nidulans. Nat. Chem. Biol. 3, 213-217. doi: 10.1038/nchembio869

Blin, K., Medema, M. H., Kazempour, D., Fischbach, M. A., Breitling, R., Takano, E., et al. (2013). antiSMASH 2.0-a versatile platform for genome mining of secondary metabolite producers. Nucleic Acids Res. 41, W204-W212. doi: $10.1093 /$ nar/gkt449

Boddy, C. N. (2014). Bioinformatics tools for genome mining of polyketide and non-ribosomal peptides. J. Ind. Microbiol. Biotechnol. 41, 443-450. doi: 10.1007/s10295-013-1368-1

Bode, H. B., Bethe, B., Höfs, R., and Zeeck, A. (2002). Big effects from small changes: possible ways to explore nature's chemical diversity. Chembiochem 3, 619-627. doi: 10.1002/1439-7633(20020703)3:7<619::AID-CBIC619>3. $0 . \mathrm{CO} ; 2-9$

Bok, J. W., Ye, R., Clevenger, K. D., Mead, D., Wagner, M., Krerowicz, A., et al. (2015). Fungal artificial chromosomes for mining of the fungal secondary metabolome. BMC Genomics 16:343. doi: 10.1186/s12864-015-1561-x

Brakhage, A. A., and Schroeckh, V. (2011). Fungal secondary metabolites strategies to activate silent gene clusters. Fungal Genet. Biol. 48, 15-22. doi: 10.1016/j.fgb.2010.04.004

Brakhage, A. A., Schuemann, J., Bergmann, S., Scherlach, K., Schroeckh, V., and Hertweck, C. (2008). Activation of fungal silent gene clusters: a new avenue to drug discovery. Prog. Drug Res. 66, 3-12. doi: 10.1007/978-3-7643-8595-8_1

Bromann, K., Toivari, M., Viljanen, K., Vuoristo, A., Ruohonen, L., and Nakari-Setala, T. (2012). Identification and characterization of a novel diterpene gene cluster in Aspergillus nidulans. PLoS ONE 7:e35450. doi: 10.1371/journal.pone.0035450

Carlson, R. (2009). The changing economics of DNA synthesis. Nat. Biotechnol. 27, 1091-1094. doi: 10.1038/nbt1209-1091

Charlop-Powers, Z., Milshteyn, A., and Brady, S. F. (2014). Metagenomic small molecule discovery methods. Curr. Opin. Microbiol. 19, 70-75. doi: 10.1016/j.mib.2014.05.021

Chiang, Y. M., Chang, S. L., Oakley, B. R., and Wang, C. C. (2014). Recent advances in awakening silent biosynthetic gene clusters and linking orphan clusters to natural products in microorganisms. Curr. Opin. Chem. Biol. 15, 137-143. doi: 10.1016/j.cbpa.2010.10.011

Chiang, Y. M., Oakley, C. E., Ahuja, M., Entwistle, R., Schultz, A., Chang, S. L., et al. (2013). An efficient system for heterologous expression of secondary metabolite genes in Aspergillus nidulans. J. Am. Chem. Soc. 135, 7720-7731. doi: $10.1021 /$ ja401945a

Chung, Y. M., Wei, C. K., Chuang, D. W., El-Shazly, M., Hsieh, C. T., Asai, T., et al. (2013). An epigenetic modifier enhances the production of anti-diabetic and anti-inflammatory sesquiterpenoids from Aspergillus sydowii. Bioorg. Med. Chem. 21, 3866-3872. doi: 10.1016/j.bmc.2013.04.004

Demain, A. L. (2014). Importance of microbial natural products and the need to revitalize their discovery. J. Ind. Microbiol. Biotechnol. 41, 185-201. doi: 10.1007/s10295-013-1325-Z

Fedorova, N. D., Moktali, V., and Medema, M. H. (2012). Bioinformatics approaches and software for detection of secondary metabolic gene clusters. Methods Mol. Biol. 944, 23-45. doi: 10.1007/978-1-62703-122-6_2

Figueroa, L., Jiménez, C., Rodríguez, J., Areche, C., Chávez, R., Henríquez, M., et al. (2015). 3-Nitroasterric acid derivatives from an Antarctic spongederived Pseudogymnoascus sp. fungus. J. Nat. Prod. 78, 919-923. doi: 10.1021/np500906k

Gross, H., Stockwell, V. O., Henkels, M. D., Nowak-Thompson, B., Loper, J. E., and Gerwick, W. H. (2007). The genomisotopic approach: a systematic method to isolate products of orphan biosynthetic gene clusters. Chem. Biol. 14, 53-63. doi: 10.1016/j.chembiol.2006.11.007

Gunde-Cimerman, N., and Zalar, P. (2014). Extremely halotolerant and halophilic fungi inhabit brine in solar salterns around the globe. Food Technol. Biotechnol. $52,170-179$.

Henrikson, J. C., Hoover, A. R., Joyner, P. M., and Cichewicz, R. H. (2009). A chemical epigenetics approach for engineering the in situ biosynthesis of a cryptic natural product from Aspergillus niger. Org. Biomol. Chem. 7, 435-438. doi: 10.1039/B819208A

Hertweck, C. (2009). Hidden biosynthetic treasures brought to light. Nat. Chem. Biol. 5, 450-452. doi: 10.1038/nchembio0709-450

Hewage, R. T., Aree, T., Mahidol, C., Ruchirawat, S., and Kittakoop, P. (2014). One strain-many compounds (OSMAC) method for production of polyketides, azaphilones, and an isochromanone using the endophytic fungus Dothideomycete sp. Phytochemistry 108, 87-94. doi: 10.1016/j.phytochem.2014.09.013

Jiao, J. Y., Wang, H. X., Zeng, Y., and Shen, Y. M. (2006). Enrichment for microbes living in association with plant tissues. J. Appl. Microbiol. 100, 830-837. doi: 10.1111/j.1365-2672.2006.02830.x

Kakule, T. B., Jadulco, R. C., Koch, M., Janso, J. E., Barrows, L. R., and Schmidt, E. W. (2015). Native promoter strategy for high-yielding synthesis and engineering of fungal secondary metabolites. ACS Synth. Biol. 4, 625-633. doi: $10.1021 / \mathrm{sb} 500296 \mathrm{p}$

Keller, N. P., Turner, G., and Bennett, J. W. (2005). Fungal secondary metabolismfrom biochemistry to genomics. Nat. Rev. Microbiol. 3, 937-947. doi: $10.1038 /$ nrmicro1286

Khaldi, N., Seifuddin, F. T., Turner, G., Haft, D., Nierman, W. C., Wolfe, K. H., et al. (2010). SMURF: genomic mapping of fungal secondary metabolite clusters. Fungal Genet. Biol. 47, 736-741. doi: 10.1016/j.fgb.2010.06.003

Larsen, T. O., Smedsgaard, J., Nielsen, K. F., Hansen, M. E., and Frisvad, J. C. (2005). Phenotypic taxonomy and metabolite profiling in microbial drug discovery. Nat. Prod. Rep. 22, 672-695. doi: 10.1039/b404943h

Lazarus, C. M., Williams, K., and Bailey, A. M. (2014). Reconstructing fungal natural product biosynthetic pathways. Nat. Prod. Rep. 31, 1339-1347. doi: 10.1039/C4NP00084F

Li, K., Chung-Davidson, Y.-W., Bussy, U., and Li, W. (2015). Recent advances and applications of experimental technologies in marine natural product research. Mar. Drugs 13, 2694-2713. doi: 10.3390/md13052694

Lin, J. Q., Zhao, X. X., Zhi, Q. Q., Zhao, M., and He, Z. M. (2013). Transcriptomic profiling of Aspergillus flavus in response to 5-azacytidine. Fungal Genet. Biol. 56, 78-86. doi: 10.1016/j.fgb.2013.04.007

Luo, Y., Cobb, R. E., and Zhao, H. (2014). Recent advances in natural product discovery. Curr. Opin. Biotechnol. 30, 230-237. doi: 10.1016/j.copbio.2014.09.002

Magnuson, J. K., and Lasure, L. L. (2002). Fungal Diversity in Soils as Assessed by Direct Culture and Molecular Techniques. (Salt Lake: Abstracts from the 102nd General Meeting of the American Society for Microbiology), 19-23. Available at: http://www.pnnl.gov/biobased/docs/fungaldiversity.pdf

Molinski, T. F. (2010). Microscale methodology for structure elucidation of natural products. Curr. Opin. Biotechnol. 21, 819-826. doi: 10.1016/j.copbio.2010.09.003

Newman, D. J., and Cragg, G. M. (2012). Natural products as sources of new drugs over the 30 years from 1981 to 2010. J. Nat. Prod. 75, 311-335. doi: $10.1021 / \mathrm{np} 200906 \mathrm{~s}$

Nikolouli, K., and Mossialos, D. (2011). Bioactive compounds synthesized by non-ribosomal peptide synthetases and type-I polyketide synthases discovered through genome-mining and metagenomics. Biotechnol. Lett. 34, 1393-1403. doi: 10.1007/s10529-012-0919-2

Niu, S., Liu, D., Hu, X., Proksch, P., Shao, Z., and Lin, W. (2014). Spiromastixones A-O, antibacterial chlorodepsidones from a deep-sea-derived Spiromastix sp. fungus. J. Nat. Prod. 77, 1021-1030. doi: 10.1021/np5000457

Nosanchuk, J. D., and Casadevall, A. (2003). The contribution of melanin to microbial pathogenesis. Cell. Microbiol. 5, 203-223. doi: 10.1046/j.14625814.2003.00268.x

Nováková, J., and Farkašovskỳ, M. (2013). Bioprospecting microbial metagenome for natural products. Biologia 68, 1079-1086. doi: 10.2478/s11756-013-0246-7

Omura, S., Ikeda, H., Ishikawa, J., Hanamoto, A., Takahashi, C., Shinose, M. et al. (2001). Genome sequence of an industrial microorganism Streptomyces 
avermitilis: deducing the ability of producing secondary metabolites. Proc. Natl. Acad. Sci. U.S.A. 98, 12215-12220. doi: 10.1073/pnas.211433198

Owen, J. G., Charlop-Powers, Z., Smith, A. G., Ternei, M. A., Calle, P. Y., Reddy, B. V., et al. (2015). Multiplexed metagenome mining using short DNA sequence tags facilitates targeted discovery of epoxyketone proteasome inhibitors. Proc. Natl. Acad. Sci. U.S.A. 112, 4221-4226. doi: 10.1073/pnas.1501124112

Reyes-Domínguez, Y., Bok, J. W., Berger, H., Shwab, E. K., Basheer, A., Gallmetzer, A., et al. (2010). Heterochromatic marks are associated with the repression of secondary metabolism clusters in Aspergillus nidulans. Mol. Microbiol. 76, 1376-1386. doi: 10.1111/j.1365-2958.2010.07051.x

Richter, L., Wanka, F., Boecker, S., Storm, D., Kurt, T., Vural, Ö., et al. (2014). Engineering of Aspergillus niger for the production of secondary metabolites. Fungal Biol. Biotechnol. 1, 4. doi: 10.1186/s40694-014-0004-9

Scherlach, K., and Hertweck, C. (2006). Discovery of aspoquinolones A-D, prenylated quinoline-2-one alkaloids from Aspergillus nidulans, motivated by genome mining. Org. Biomol. Chem. 4, 3517-3520. doi: 10.1039/ b607011f

Scherlach, K., and Hertweck, C. (2009). Triggering cryptic natural product biosynthesis in microorganisms. Org. Biomol. Chem. 7, 1753-1760. doi: $10.1039 / \mathrm{b} 821578 \mathrm{~b}$

Strauss, J., and Reyes-Domínguez, Y. (2011). Regulation of secondary metabolism by chromatin structure and epigenetic codes. Fungal Genet. Biol. 48, 62-69. doi: 10.1016/j.fgb.2010.07.009

Strohl, W. R. (2000). The role of natural products in a modern drug discovery program. Drug Discov. Today 5, 39-41. doi: 10.1016/S1359-6446(99)01443-9

Timling, I., and Taylor, D. L. (2012). Peeking through a frosty window: molecular insights into the ecology of Arctic soil fungi. Fungal Ecol. 5, 419-429. doi: 10.1016/j.funeco.2012.01.009

VanderMolen, K. M., Raja, H. A., El-Elimat, T., and Oberlies, N. H. (2013). Evaluation of culture media for the production of secondary metabolites in a natural products screening program. AMB Express 3, 71. doi: 10.1186/21910855-3-71

Wang, W.-J., Li, D.-Y., Li, Y.-C., Hua, H.-M., Ma, E.-L., and Li, Z.-L. (2014). Caryophyllene sesquiterpenes from the marine-derived fungus Ascotricha sp.
ZJ-M-5 by the one strain-many compounds strategy. J. Nat. Prod. 77, 13671371. doi: $10.1021 / \mathrm{np} 500110 \mathrm{z}$

Weber, T., Blin, K., Duddela, S., Krug, D., Kim, H. U., Bruccoleri, R., et al. (2015). antiSMASH 3.0-a comprehensive resource for the genome mining of biosynthetic gene clusters. Nucleic Acids Res. 43, W237-W243. doi: 10.1093/nar/gkv437

Wiemann, P., and Keller, N. P. (2014). Strategies for mining fungal natural products. J. Ind. Microbiol. Biotechnol. 41, 301-313. doi: 10.1007/s10295-0131366-3

Williams, R. B., Henrikson, J. C., Hoover, A. R., Lee, A. E., and Cichewicz, R. H. (2008). Epigenetic remodeling of the fungal secondary metabolome. Org. Biomol. Chem. 6, 1895-1897. doi: 10.1039/b804701d

Wilson, M. C., and Piel, J. (2013). Metagenomic approaches for exploiting uncultivated bacteria as a resource for novel biosynthetic enzymology. Chem. Biol. 20, 636-647. doi: 10.1016/j.chembiol.2013.04.011

Woodhouse, J. N., Fan, L., Brown, M. V., Thomas, T., and Neilan, B. A. (2013). Deep sequencing of non-ribosomal peptide synthetases and polyketide synthases from the microbiomes of Australian marine sponges. ISME J. 7, 1842-1851. doi: 10.1038/ismej.2013.65

Yin, W. B., Chooi, Y. H., Smith, A. R., Cacho, R. A., Hu, Y., White, T. C., et al. (2013). Discovery of cryptic polyketide metabolites from dermatophytes using heterologous expression in Aspergillus nidulans. ACS Synth. Biol. 2, 629-634. doi: $10.1021 / \mathrm{sb} 400048 \mathrm{~b}$

Conflict of Interest Statement: The authors declare that the research was conducted in the absence of any commercial or financial relationships that could be construed as a potential conflict of interest.

Copyright (c) 2015 Chávez, Fierro, García-Rico and Vaca. This is an open-access article distributed under the terms of the Creative Commons Attribution License (CC BY). The use, distribution or reproduction in other forums is permitted, provided the original author(s) or licensor are credited and that the original publication in this journal is cited, in accordance with accepted academic practice. No use, distribution or reproduction is permitted which does not comply with these terms. 